\title{
FORCED AND VOLUNTARY EXERCISE DIFFERENTIALLY AFFECT BRAIN AND BEHAVIOR
}

\author{
J. L. LEASURE ${ }^{a, b *}$ AND M. JONES ${ }^{a}$ \\ a Department of Psychology, 126 Heyne Building, University of Hous- \\ ton, Houston, TX 77204, USA \\ ${ }^{b}$ Department of Biology and Biochemistry, University of Houston, \\ Houston, TX 77204, USA
}

\begin{abstract}
The potential of physical exercise to decrease body weight, alleviate depression, combat aging and enhance cognition has been well-supported by research studies. However, exercise regimens vary widely across experiments, raising the question of whether there is an optimal form, intensity and duration of exertion that would produce maximal benefits. In particular, a comparison of forced and voluntary exercise is needed, since the results of several prior studies suggest that they may differentially affect brain and behavior. In the present study, we employed a novel 8-week exercise paradigm that standardized the distance, pattern, equipment and housing condition of forced and voluntary exercisers. Exercising rats were then compared with sedentary controls on measures previously shown to be influenced by physical activity. Our results indicate that although the distance covered by both exercise groups was the same, voluntary exercisers ran at higher speed and for less total time than forced exercisers. When compared with sedentary controls, forced but not voluntary exercise was found to increase anxiety-like behaviors in the open field. Both forms of exercise increased the number of surviving bromodeoxyuridine (BrdU)+ cells in the dentate gyrus after 8 weeks of exercise, although forced exercisers had significantly more than voluntary exercisers. Phenotypic analysis of BrdU+ cells showed no difference between groups in the percentage of newborn cells that became neurons, however, because forced exercise maximally increased the number of BrdU+ cells, it ultimately produced more neurons than voluntary exercise. Our results indicate that forced and voluntary exercise are inherently different: voluntary wheel running is characterized by rapid pace and short duration, whereas forced exercise involves a slower, more consistent pace for longer periods of time. This basic difference between the two forms of exercise is likely responsible for their differential effects on brain and behavior. (C) 2008 IBRO. Published by Elsevier Ltd. All rights reserved.
\end{abstract}

Key words: voluntary, forced, anxiety, water maze, dentate gyrus, progenitor cells.

Although evidence of the neural and behavioral benefits of exercise is accumulating, the optimal type, duration and

${ }^{*}$ Correspondence to: J. L. Leasure, Department of Psychology, 126 Heyne Building, University of Houston, Houston, TX 77204, USA. Tel: +1-713-743-8616; fax: +1-713-743-8588.

E-mail address: j.leasure@mail.uh.edu (J. L. Leasure).

Abbreviations: BrdU, bromodeoxyuridine; HPA, hypothalamic-pituitary-adrenal axis; NIH, National Institutes of Health; TBS, tris-buffered saline; TBST, $0.3 \%$ tris-buffered saline/Triton-X.

0306-4522/08 @ 2008 IBRO. Published by Elsevier Ltd. All rights reserved. doi:10.1016/j.neuroscience.2008.07.041 intensity of long-term physical activity have not been established. Laboratory animal models of chronic exercise are highly variable, with many studies utilizing ad libitum access to voluntary exercise wheels, while others employ comparably short bouts of forced exercise on a treadmill. Not surprisingly, studies of the same outcome measure produce different results, depending on whether a voluntary or forced exercise paradigm is used (for a recent review of these disparities, see Ang and Gomez-Pinilla, 2007). In addition, it is becoming increasingly recognized that no single exercise paradigm is likely to fulfill all therapeutic needs (Ang and Gomez-Pinilla, 2007; Cotman et al., 2007). Thus, it is imperative to study the neural and behavioral effects of different forms of chronic exercise while holding their parameters constant.

Human studies have attempted to pinpoint the optimal intensity level at which acute exercise maximally benefits cognition (Winter et al., 2007; McMorris et al., 2008, see Brisswalter et al., 2002 for a useful review), event-related brain potentials (ERP's) (Kamijo et al., 2004b, 2007) and arousal level (Kamijo et al., 2004a). The most beneficial intensity, duration and type of long-term physical activity have not been well-studied in laboratory animals, however. The type of exercise may be particularly important, since several lines of evidence suggest that forced exercise and voluntary exercise exert different effects on the brain and behavior. For example, forced and voluntary exercise differentially affect monoamine neurotransmitters (Dishman, 1997), hippocampal parvalbumin expression (Arida et al., 2004), hippocampal brain-derived neurotrophic factor and synapsin-1 expression (Ploughman et al., 2005), longevity and body composition (Narath et al., 2001), taste aversion learning (Masaki and Nakajima, 2006) and open-field behavior (Burghardt et al., 2004). Although these studies suggest that forced and voluntary exercise may not be equivalent in their effects on the brain and behavior, a carefully matched comparison has never been made. In fact, with a few notable exceptions (Dishman, 1997; Dishman et al., 1997; Noble et al., 1999; Greenwood et al., 2003; Burghardt et al., 2004; Ploughman et al., 2005) the two forms of exercise are often treated as if they are equivalent. Even studies that make use of both forced and voluntary exercise paradigms do not attempt to match the distance, duration or intensity of exercise between groups. To illustrate, forced exercisers are usually required to cover only short distances. Mice and rats will voluntarily run long distances (see for example Rodnick et al., 1989; Lambert et al., 1996; Allen et al., 2001; Burghardt et al., 2004; Naylor et al., 2005), yet many studies that use forced exercise paradigms run the animals for only a few hundred 

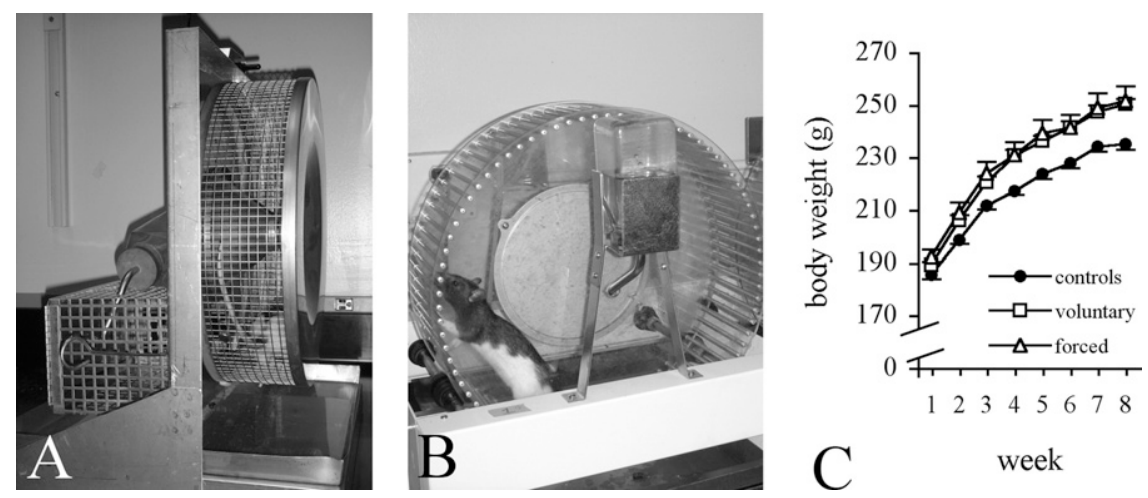

Fig. 1. Rats in the voluntary condition (A) exercised in freely moving wheels, while rats in the forced condition (B) had to maintain the pace of motorized wheels. All rats gained weight during the course of the experiment (C).

meters (Carro et al., 2000; Kim et al., 2002; Ra et al., 2002; Burghardt et al., 2004). Such studies typically also restrict forced runners to about an hour on the treadmill, once or perhaps twice a day, 5 days per week, while allowing voluntary runners $24-\mathrm{h}$ access to running wheels. Thus, in most studies, neither the distance covered nor the time spent running by forced exercisers approximates that of spontaneous exercisers.

In the current study we employed a novel exercise paradigm to test the hypothesis that equivalent amounts of forced and voluntary exercise would exert different effects on physiological, behavioral and neural parameters previously shown to be influenced by exercise, including body weight (Lambert et al., 1996; Moraska et al., 2000; Droste et al., 2007), abdominal fat (Droste et al., 2007), hind-limb musculature (Rodnick et al., 1989), spatial navigation (Fordyce and Farrar, 1991a; van Praag et al., 1999a; Vaynman et al., 2004; Ang et al., 2006), open-field activity (Burghardt et al., 2004; Fulk et al., 2004) and survival and differentiation of hippocampal progenitor cells (van Praag et al., 1999a).

\section{EXPERIMENTAL PROCEDURES}

\section{Animals}

All experimental procedures were conducted in accordance with the National Institutes of Health (NIH) Public Health Service Policy on Humane Care and Use of Laboratory Animals (NIH 2002), and were approved by the Institutional Animal Care and Use Committee of the University of Houston. Furthermore, animal suffering was minimized where possible and the lowest possible number of animals was used. Thirty-four female Long-Evans rats (Harlan Sprague Dawley, Indianapolis, IN, USA), weighing 170-200 g at the beginning of the experiment were divided into three groups; sedentary controls $(n=12)$, voluntary runners $(n=13)$ and forced runners $(n=9)$. When not exercising, rats were housed in groups of three, on a reversed 12-h light/dark cycle $(10: 00 \mathrm{~h}$ off/22:00 h on) with food and water available ad libitum.

\section{Novel exercise paradigm matching distance of voluntary and forced exercise}

Rats were exercised daily, 5 days per week, for 8 weeks, beginning at the start of the dark cycle $(10: 00 \mathrm{~h})$. In order to directly compare the effects of voluntary and forced exercise on body and brain, it was necessary to both limit the distance covered by the voluntary exercisers and lengthen the distance run by the forced exercisers. We limited the distance run by the voluntary exercisers by allowing access to a wheel (see Fig. 1A) only until each rat reached a daily maximum, which was increased weekly during the 8-week exercise period, beginning with approximately $1300 \mathrm{~m}$ in Week 1 and gradually increasing to $2300 \mathrm{~m}$ in Week 8 . The voluntary exercise wheels were equipped with counters that recorded distance traveled, average speed and time spent running. The distance covered by the forced exercisers was matched daily to the average distance run by the voluntary exercisers. The sedentary control rats were placed into immobilized wheels that were otherwise identical to those used by the voluntary runners. All rats had access to water at all times during the exercise period.

We employed four general strategies to standardize the exercise conditions of the forced and voluntary runners. First, we chose not to use a treadmill. Instead, forced exercisers ran in motorized wheels atop rotating axles (Lafayette Instruments, Lafayette, IN, USA) (see Fig. 1B). This strategy ensured that both groups were running in wheels (rather than the forced exercisers running on a belt), and that those wheels were similarly sized. In addition, it ensured that our forced exercisers were not exposed to aversive electric shocks, since commercially available treadmills (but not our motorized apparatus) have an electric shock component that can be used to "encourage" the animals to maintain the pace of the treadmill belt.

Second, the forced exercisers ran in a pattern consistent with the manner in which rats exercise voluntarily-in short bursts of activity interspersed with frequent rests (Eikelboom and Mills, 1988). The motorized wheel apparatus was controlled by a keypad that allowed the speed and duration of exercise, as well as periods of rest, to be pre-set. For example, the motorized axles could be programmed to turn the wheels at $15 \mathrm{~m} / \mathrm{min}$ for $40 \mathrm{~s}$, followed by a $20 \mathrm{~s}$ rest period, during which the wheels stopped turning. This sequence represented a single cycle. The cycle was repeated, and combined with additional cycles of faster or slower speeds $(5-30 \mathrm{~m} / \mathrm{min})$ until the forced runners had reached the average distance run by the voluntary runners for the day. Thus, the forced exercisers were not running constantly for a fixed amount of time, as they usually are in treadmill exercise studies. We found that rats were reluctant to run at speeds higher than 20 $\mathrm{m} / \mathrm{min}$, therefore, higher speeds were used sparingly and for short durations. Rats were observed carefully to ensure that they kept pace with the wheels. One rat was eliminated from the study because it held onto the spokes of the wheel through the entire rotation.

Third, each day the distance covered by the forced exercisers was matched to the average distance run by the voluntary exercisers on that day. This ensured that both groups ran similar distances daily throughout the course of the experiment (see Fig. $2 A)$. The distance that the voluntary exercisers were allowed to 

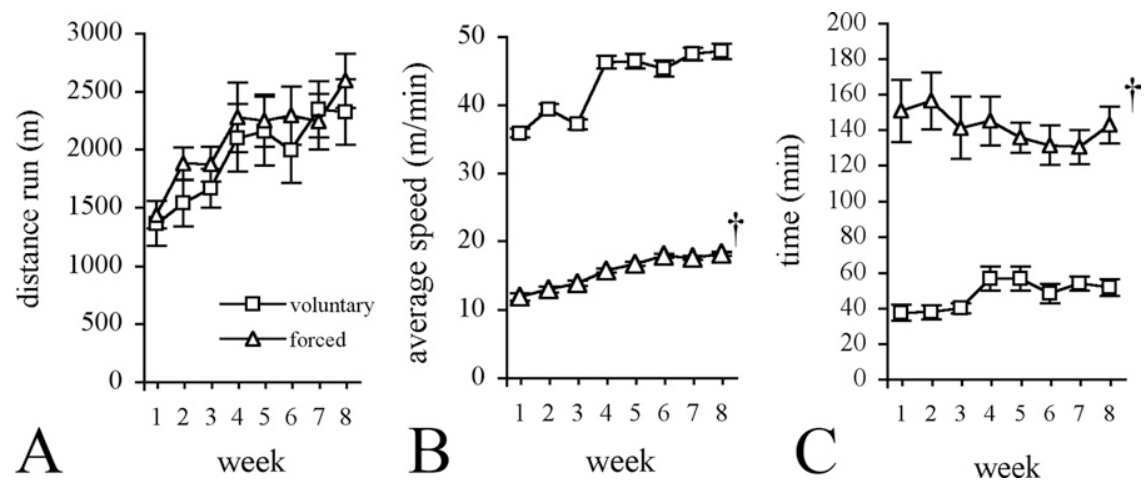

Fig. 2. The activity of the voluntary and forced runners was matched so that there was no significant difference between exercise groups for distance traveled (A). The voluntary exercisers ran significantly faster $(B)$ and for a significantly shorter time than the forced exercisers $(C)$. ${ }^{\dagger} P<0.05$ significantly different from voluntary exercisers.

run was progressively increased over the 8-week course of the experiment, because rats allowed long-term ad libitum access to running wheels gradually increase the distance covered during the initial 4 to 8-week period (Mondon et al., 1985; Rodnick et al., 1989; Lambert et al., 1996; Droste et al., 2007).

Fourth, all animals in the present study were housed in groups of three, and isolated only during the time when they were exercising. Removing the animals from the home cage only during exercise made it unnecessary to permanently single-house each voluntary runner with an exercise wheel, and ensured that animals in all groups could be maintained in social housing, which is more compatible with the rat's gregarious nature than isolated housing.

\section{Bromodeoxyuridine (BrdU) injections}

BrdU (Sigma, St. Louis, MO, USA), a thymidine analog, was administered daily for 5 days during the fifth week of exercise. Each day, immediately prior to exercise, each animal was weighed and then injected with $50 \mathrm{mg} / \mathrm{kg}$ of $\mathrm{BrdU}$, i.p.

\section{Fecal pellet observation and collection for corticosterone analysis}

The rodent stress hormone, corticosterone, can be accurately measured from fecal samples (Cavigelli et al., 2005; Thanos et al., in press). Fecal corticosterone levels are tightly correlated with serum corticosterone levels (Thanos et al., in press), making fecal sampling advantageous because it is not affected by acute changes in hormone levels due to invasive sampling procedures necessary for blood collection via tail vein or jugular cannula. Furthermore, fecal corticosterone analysis is ideal for measuring the long-term corticosterone response to an ongoing experimental manipulation (Thanos et al., in press), such as chronic exercise. We collected fecal pellets from a subset of rats in the present study at noon on day 36 (week 8) of exercise. Collection of fecal pellets was simple, since we were able to obtain a fresh pellet from the pan under the wheel of each rat, including the sedentary controls, which were in immobilized wheels. Care was taken to ensure that the pellets had not contacted urine puddles, since urine contains less corticosterone than feces (Bamberg et al., 2001) and urine contamination can cause corticosterone to leach out of fecal pellets. After collection, pellets were stored at $-80^{\circ} \mathrm{C}$ until they were sent for analysis via competitive enzyme immunoassay (Cayman Chemical, Ann Arbor, MI, USA).

In the process of collecting fecal pellets, we noticed that there were many more pellets available under the wheels of the forced exercisers, compared with the voluntary runners and sedentary controls. Defecation has been used as a measure of emotionality in rats (Hall, 1934; Sanberg, 1989), so we counted the number of fecal pellets deposited by rats in each group during the first hour of exercise on the last (40th) day of exercise.

\section{Analysis of spatial learning}

Each day during the eighth week of exercise, prior to the start of exercise, all rats were tested in the Morris water maze $(167 \mathrm{~cm}$ diameter, $76 \mathrm{~cm}$ deep), using a difficult two-trial-per-day paradigm that has been shown to detect subtle differences in performance of mice (van Praag et al., 1999a) and rats (Vaynman et al., 2004). Each rat was given two trials per day for five consecutive days. The location of the platform remained constant throughout testing but the release point changed each day. Rats were released facing the wall, from one of four randomly chosen points, and allowed $60 \mathrm{~s}$ to find the hidden platform. The surface of the water was made opaque by the addition of tiny Styrofoam beads. If the rat failed to find the platform in $60 \mathrm{~s}$, it was removed from the water, placed on the platform and allowed to remain there for $10 \mathrm{~s}$. If the rat did find the platform, it was allowed to remain there for $10 \mathrm{~s}$, then removed from the maze and dried with a towel. Extramaze reference cues included posters, doorways and a shelving unit. Latency to locate the platform, swim speed and swim path were recorded by an automated tracking system (Noldus, Amsterdam, Netherlands). Memory retention was tested with a probe trial $24 \mathrm{~h}$ after the last training trial.

\section{Analysis of exploratory behavior in the open field}

On the last day of exercise, after water maze training and prior to beginning the daily exercise regimen, each rat was tested in the open field, which consisted of a plywood box with a white plastic bottom divided into 25 equally-sized squares (each $15 \times 15 \mathrm{~cm}$ ). Each rat was placed in the center of the open field and videorecorded for $5 \mathrm{~min}$. The following outcome measures were assessed: number of outer squares entered, number of inner squares entered, time spent in the outer squares, time spent in the inner squares and the number of rears (standing upright, supported by hind limbs) (Burghardt et al., 2004). The video footage of one forced exerciser was lost due to mechanical failure.

\section{Adrenal gland, abdominal fat deposit and gastrocnemius muscle weights}

The day after the final (40th) day of exercise, each rat was overdosed with anesthetic, the body cavity opened, and adrenal glands and the i.p. fat deposit (Droste et al., 2007) rapidly collected and weighed. An index score was calculated for the adrenal glands of each animal: (adrenal gland weight $(\mathrm{mg}) / \mathrm{body}$ weight (g) $) \times 100$ (Lambert et al., 1998). The right hind limb was removed 
from a subset of animals ( $n=18$, six per group) for collection and weighing of the gastrocnemius muscle. Abdominal fat and hindlimb muscle was collected because we wanted to assess whether body fat had decreased and muscle mass increased in the exercising rats. Each animal was then intracardially perfused with cold saline, followed by $4 \%$ paraformaldehyde, until the upper body was stiff.

\section{Histological procedures}

Brains were removed and post-fixed overnight, followed by refrigeration in $30 \%$ sucrose. Brains were cut in $40 \mu \mathrm{m}$ sections on a Vibratome and stored in cryoprotectant in 96-well microtiter plates at $-20^{\circ} \mathrm{C}$. The brain of one sedentary control animal was irreparably damaged during processing, and could not be included in histological analysis.

BrdU peroxidase labeling was performed on every 12 th section by washing them in tris-buffered saline (TBS) followed by treatment with $0.3 \%$ hydrogen peroxide for $30 \mathrm{~min}$ to exhaust endogenous peroxidases. Sections were then washed with $0.3 \%$ tris-buffered saline/Triton-X (TBST) three times at $10 \mathrm{~min}$ each. DNA was denatured by treating the sections with $2 \mathrm{~N} \mathrm{HCl}$ for 30 min at $37^{\circ} \mathrm{C}$, followed by a 10 min neutralization with $0.1 \mathrm{M}$ borate. After rinsing with TBST and blocking for $2 \mathrm{~h}$ in 10\% donkey serum, sections were incubated overnight $\left(4^{\circ} \mathrm{C}\right)$ with primary antibody (sheep anti-BrdU, Exalpha Biologicals, Maynard, MA, USA, 1:400). After three rinses in TBS, sections were incubated with secondary antibody (biotinylated donkey anti-sheep, Jackson Laboratories, West Grove, PA, USA, 1:250) for $2 \mathrm{~h}$ at room temperature, rinsed again and then incubated with avidin-biotin complex (ABC, Vector Laboratories, Burlingame, CA, USA) for $1 \mathrm{~h}$. The final step was incubation with diaminobenzidine (DAB) and $0.0006 \%$ hydrogen peroxide in TBS followed by mounting and coverslipping.

For triple immunofluorescent labeling for BrdU, NeuN and Iba1, every 12th section was treated as described above, except that the initial hydrogen peroxide treatment was omitted. After the blocking step, sections were incubated in primary antibodies (mouse anti-NeuN, Chemicon, Temecula, CA, USA, 1:20,000; sheep anti-BrdU, Exalpha Biologicals, 1:1000; rabbit anti-lba1, Wako, Japan, $1: 15,000$ ) for $72 \mathrm{~h}$ at $4{ }^{\circ} \mathrm{C}$. Sections were rinsed and then incubated in secondary antibodies (FITC-conjugated donkey anti-mouse, CY3-conjugated donkey anti-sheep, CY5-conjugated donkey anti-rabbit, all from Jackson Laboratories, 1:250).

\section{Quantification of BrdU+ cells}

Coded slides were viewed with a $40 \times$ objective using a Nikon Eclipse 80i upright microscope equipped with an automated stereology system, Stereolnvestigator (MicroBrightField, Williston, VT, USA). As previously described (Redila et al., 2006), each $\mathrm{BrdU}+$ cell located in the granule cell layer (GCL) and subgranular zone (SGZ, defined as zero to two cell bodies from the inner molecular layer) was counted in every 12 th section throughout the rostro-caudal extent of the dentate gyrus. We used a modified optical dissector method such that cells that appeared in focus in the uppermost focal plane were not counted (Kempermann et al., 2003). Reference volume was determined in Cresyl Violet-stained serial sections (each $240 \mu \mathrm{m}$ apart) using the Cavalieri estimator, applied via Stereolnvestigator. The coefficient of error was no more than 0.01 for each volume estimate.

\section{Phenotype analysis}

In order to determine the phenotype of newborn cells, $50 \mathrm{BrdU}+$ cells in the dentate gyrus of each animal were examined for coexpression with either NeuN (neurons) or Iba1 (microglia). Fluorescent signals from sections on coded slides were viewed using a confocal microscope (Olympus Fluoview 1000) with a $60 \times \mathrm{PL}$
APO oil immersion objective (NA 1.42). Each potentially doublelabeled cell was examined in a Z-stack obtained at $60 \times$ and stepped through $1 \mu \mathrm{m}$ at a time, in order to verify coexpression of markers. Sequential scanning was employed in order to minimize non-specific signal crossover between channels.

\section{Statistical analyses}

All values presented are expressed as mean \pm standard error of the mean. Running distance, body weight and water maze data were analyzed using repeated measures ANOVA. All other data were analyzed using factorial ANOVA. Pair-wise post hoc comparisons were conducted when necessary using Fisher's PLSD. For all statistical analyses, a $P$ value of less than 0.05 was deemed significant.

\section{RESULTS}

\section{Body weight, distance traveled, average speed and time spent running}

ANOVA revealed no significant effect of treatment condition on body weight $(F(2,31)=3.13, P>0.05)$. All groups gained a significant amount of weight during the 8-week course of the experiment $(F(7,14)=228.29, \quad P<0.05)$. There was no significant difference between the distance run by voluntary and forced exercisers $(F(1,20)=0.78$, $P>0.05)$, but both groups significantly increased the distance run over time $(F(7,7)=15.45, P<0.05)$ (Fig. 2A). In contrast, the average speed of the voluntary runners was considerably faster than that of the forced exercisers $(F(1,17)=390.00, P<0.05)$ (Fig. 2B). The average speed of both groups significantly increased during the course of the experiment $(F(7,119)=21.92, P<0.05)$. There was a significant difference between groups for time spent running $(F(1,17)=80.08, P<0.05)$ but neither group significantly increased the time spent running during the course of the study $(F(7,119)=0.61, P>0.05)$.

\section{Open-field behaviors and emotional defecation}

Table 1 contains the results of the open field test. ANOVA revealed a significant effect of treatment condition for total number of squares entered $(F(2,30)=3.42, P<0.05)$, and post hoc analysis showed that forced exercisers entered significantly fewer total squares compared with sedentary controls $(P=0.01)$. There was no significant difference between groups for total number of outer squares entered $(F(2,30)=1.51, P>0.05)$. However, ANOVA indicated a significant difference for total number of inner squares entered $(F(2,30)=5.09, P<0.05)$ and post hoc analysis revealed that both sedentary controls and voluntary runners entered significantly more inner squares than forced runners did ( $P=0.01$ and 0.02 respectively). Furthermore, there was a significant difference between groups on amount of time spent in the inner squares $(F(2,30)=3.90$, $P<0.05)$ and post hoc analysis showed that both sedentary controls and voluntary runners spent more time in the inner squares compared with forced runners $(P=0.01$ and 0.03 respectively). There was no difference between groups on number of rears $(F(2,30)=1.174, P>0.05)$ made during exposure to the open field. 
Table 1. Behavior in the open field test

\begin{tabular}{lllll}
\hline Type of exercise & \multicolumn{2}{l}{ Squares entered } & & Time (s), inner squares \\
\cline { 2 - 4 } & Outer & Inner & Total & \\
\hline Sedentary & $140.7 \pm 6.3$ & $24.5 \pm 4.6$ & $165.2 \pm 9.5$ & $15.6 \pm 3.5$ \\
Voluntary & $129.8 \pm 5.0$ & $22.7 \pm 4.2$ & $152.5 \pm 7.0$ & $13.8 \pm 1.9$ \\
Forced & $124.1 \pm 13.1$ & $5.8 \pm 2.0^{\star \dagger}$ & $129.7 \pm 13.8^{* \dagger}$ & $4.8 \pm 1.7^{* \dagger}$
\end{tabular}

${ }^{*} P<0.05$ significantly different from sedentary control.

${ }^{\dagger} P<0.05$ significantly different from voluntary exercisers.

Table 2 shows the number of fecal pellets deposited by each group of rats on the final day of exercise. There was a significant effect of treatment condition on the number of pellets deposited $(F(2,31)=7.95, P<0.05)$. Forced exercisers deposited significantly more pellets than the sedentary controls $(P=0.001)$ or voluntary exercisers $(P=0.002)$.

\section{Water maze performance}

Repeated measures ANOVA showed that all groups learned to find the platform equally quickly. There was no effect of treatment condition $(F(2,31)=1.82, P>0.05)$, and no treatment $\times$ time interaction $(F(8,124)=0.99, P>0.05)$. There was a significant effect of time $(F(4,124)=48.13$, $P<0.05$ ), indicating that rats in all groups decreased their escape latencies over the course of testing (see Fig. 3A). There was no significant difference between groups on time spent in the platform quadrant during the probe trial $(F(2,31)=1.67, P>0.05)$ (Fig. 3B-D).

\section{Physiological and stress-related measures}

Table 2 shows the data from the physiological measures. There was no significant effect of treatment condition on adrenal gland weight $(F(2,31)=1.87, P>0.05)$, weight of the i.p. fat deposit $(F(2,30)=1.82, P>0.05)$, gastrocnemius muscle wet weight $(F(2,14)=2.66, P>0.05)$, or fecal corticosterone $(F(2,14)=0.10, P>0.05)$.

\section{Survival and differentiation of BrdU+ cells in the dentate gyrus}

The volume of the dentate gyrus did not differ between groups $(F(2,30)=1.70, P>0.05)$. However, ANOVA revealed a significant main effect of treatment condition on the number of BrdU+ cells in the dentate gyrus $(F(2,30)=8.58, P<0.05)$. Post hoc analysis showed that both voluntary and forced exercisers had more BrdU+ cells compared with sedentary controls $(P=0.03$ and 0.0003 respectively). In addition, forced exercisers had significantly more labeled cells compared with voluntary exercisers $(P=0.04)$ (Fig. 4A).

We analyzed BrdU+ cells for coexpression with the neuronal marker NeuN and found that the majority of $\mathrm{BrdU}+$ cells were also NeuN + . The percentage of BrdU+ cells that were also $\mathrm{NeuN}+$ did not differ between groups $(F(2,30)=1.33, P>0.05$; sedentary: $76.73 \pm 3.0$; voluntary: 82.46 \pm 2.3 ; forced: 81.11 \pm 2.6$)$. We also analyzed BrdU+ cells for colabeling with Iba1, a microglial marker. Although Iba1+ cells were present (see Fig. 4B), BrdU+/lba1+ cells were extremely rare and equally distributed among experimental conditions (a mean of less than one BrdU+/lba1+ cell was found per group).

\section{DISCUSSION}

The present study was designed to compare the effects of voluntary and forced exercise on body, brain and behavior. Other investigators have examined both types of exercise in a single study (for example, Dishman et al., 1996; Narath et al., 2001; Arida et al., 2004; Burghardt et al., 2004; Ploughman et al., 2005), however, we are the first to standardize the distance traveled by forced and voluntary exercisers. Our results indicate that even when distance is held constant, forced and voluntary exercise differ in several ways. First, voluntary exercisers reach much higher speeds than forced exercisers. We found that the overall average speed of our voluntary wheel-runners was 43.7 $\mathrm{m} / \mathrm{min}$, compared with $15.5 \mathrm{~m} / \mathrm{min}$ in our forced runners (Fig. 2B). Average speed is rarely reported for spontaneously exercising rats, but our findings are consistent with at least one prior study (Rodnick et al., 1989). In contrast, the reluctance of forced runners to maintain the speed of a treadmill belt or motorized wheel has often been noted (Leger and Taylor, 1982; Armstrong et al., 1983; Russell et al., 1987; Narath et al., 2001). We found it difficult to force rats to run at speeds greater than $20 \mathrm{~m} / \mathrm{min}$, which meant that their average speed was much slower than that of the

Table 2. Physiological and stress-related measures

\begin{tabular}{|c|c|c|c|c|}
\hline Type of exercise & Fecal pellets & Adrenal gland index score & Abdominal fat mass $(\mathrm{g})$ & Gastrocnemius wet weight $(\mathrm{g})$ \\
\hline Sedentary & $1.9 \pm 0.6$ & $96.5 \pm 7.5$ & $2.1 \pm 0.3$ & $1.1 \pm 0.1$ \\
\hline Voluntary & $2.4 \pm 0.6$ & $80.1 \pm 6.4$ & $1.4 \pm 0.2$ & $1.3 \pm 0.04$ \\
\hline Forced & $6.1 \pm 1.1^{* \dagger}$ & $82.5 \pm 4.1$ & $1.4 \pm 0.3$ & $1.2 \pm 0.1$ \\
\hline
\end{tabular}

* $P<0.05$ significantly different from sedentary control.

$+P<0.05$ significantly different from voluntary exercisers. 

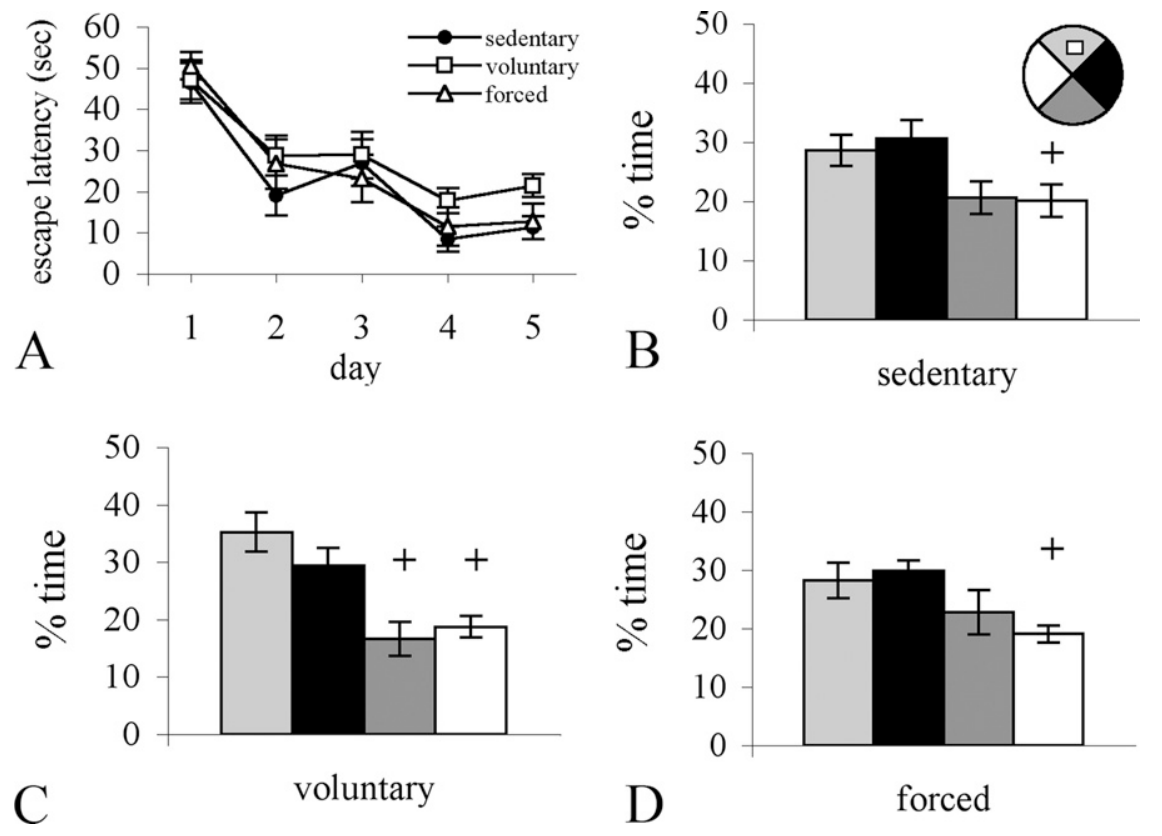

Fig. 3. Latency to escape from the water maze did not differ between groups (A). There was no difference between groups for time spent in the platform quadrant during the probe trial $(B-D) .{ }^{+} P<0.05$ significantly different from platform quadrant.

voluntary exercisers. Second, because forced exercisers go slower, it is necessary for them to run for longer periods of time if they are to match the distance covered by voluntary runners (Fig. 2C). This inherent difference in exercise regimens of varying intensity (where intensity is determined by average speed) has been noted before (Winter et al., 2007). That is, if two groups of exercisers are to achieve the same total distance, the group that travels at a faster average speed will finish in less time than one for which the average speed is slower. Finally, volition is characteristic of spontaneous wheel-running, but absent from forced paradigms. Running wheels allow rats to selfdetermine when, how often and for how long to exercise, whereas these parameters are pre-determined for forced runners who must exercise even if not motivated to do so. These inherent differences likely underlie the differential effects observed in the present study. Forced, but not voluntary exercise increased emotional defecation and
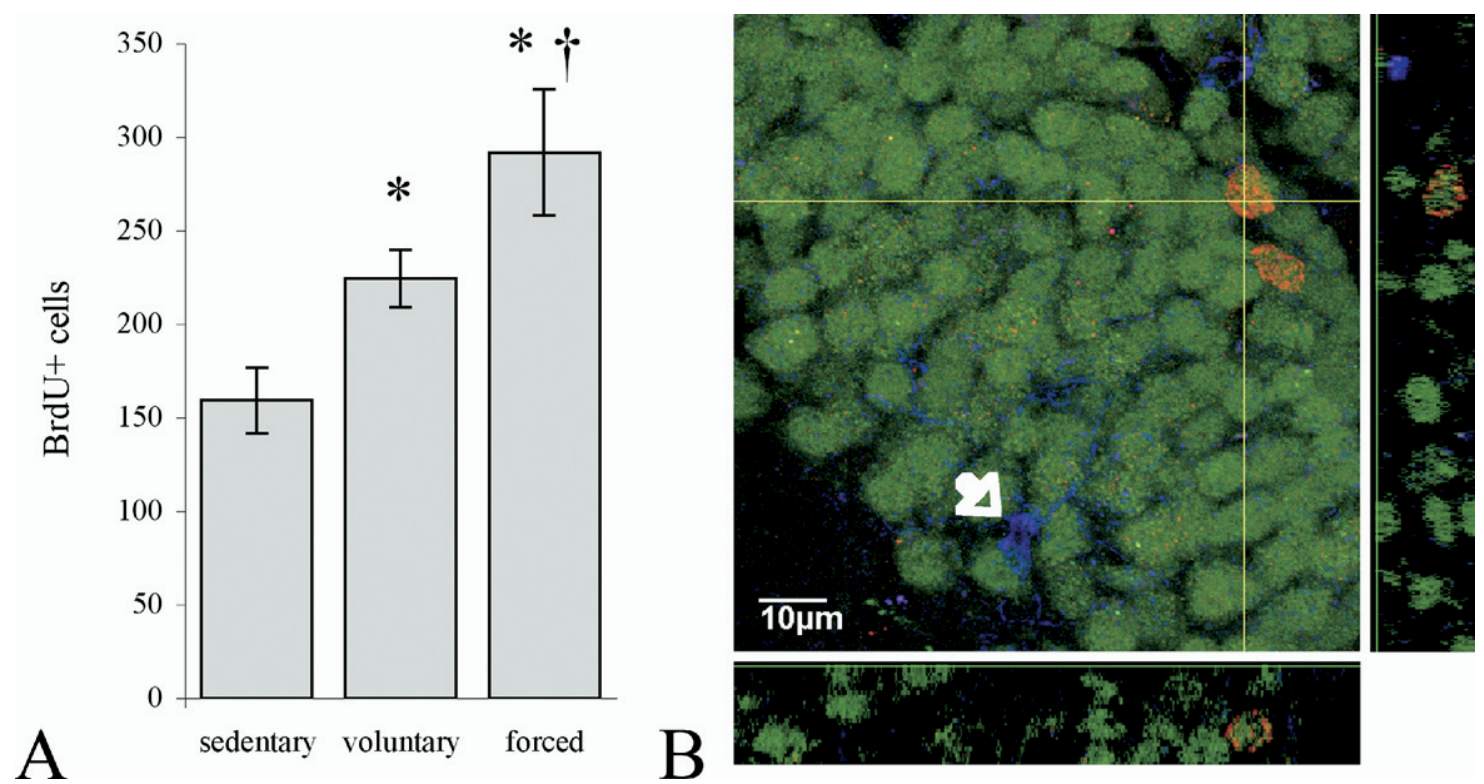

Fig. 4. Both voluntary and forced exercise increased survival of BrdU+ cells in the dentate gyrus (A). However, forced exercisers had significantly more BrdU-labeled cells in the dentate compared with voluntary exercisers. (B) $Z$ stack showing two representative BrdU $+/ \mathrm{NeuN}+$ cells, as well as an Iba1+ microglial cell (white arrow). ${ }^{*} P<0.05$ significantly different from sedentary controls, ${ }^{\dagger} P<0.05$ significantly different from voluntary exercisers. 
anxiety-like behaviors. Although both types of exercise increased survival of progenitor cells in the dentate gyrus, this effect was more marked with forced exercise. Exercise did not increase the percentage of progenitor cells that adopted a neuronal phenotype, however, because the pool of surviving progenitors was largest in the forced exercisers, this type of exercise ultimately resulted in more net neurogenesis.

In order to obtain equivalent, behaviorally relevant amounts of exercise it was necessary to both restrict our voluntary runners to shorter distances than those typically run in studies of voluntary exercise, and to make our forced runners run further than rodents in prior treadmill studies. It is difficult, therefore, to directly compare our results to the existing literature on exercise. We did not find differential effects of the two forms of exercise on any physiological measures. Weight loss has been found in some (Lambert et al., 1996; Moraska et al., 2000; Droste et al., 2007) but not other (Fordyce and Farrar, 1991b) long-term exercise studies, accompanied by decreased abdominal fat mass (Droste et al., 2007) and increased hind-limb musculature (Rodnick et al., 1989). We found no significant differences in body weight, abdominal fat or hind-limb muscle weight. We also found no differences between groups in fecal corticosterone levels or adrenal gland weight, both of which gauge chronic hypothalamic-pituitary-adrenal (HPA) axis response. Prior research suggests that the HPA axis adapts to chronic exercise (Fediuc et al., 2006), and, consistent with this idea, we found no differences between groups in fecal corticosterone levels after 8 weeks of exercise. Hypertrophy of the adrenal glands may result from chronic exercise (Moraska et al., 2000), suggesting a longterm stress response, although not all studies have shown this (Fordyce and Farrar, 1991b; Droste et al., 2007). We did not find any differences between groups in adrenal gland weight. Taken together, our results suggest that long-term exposure to restricted daily exercise does not result in an enhanced stress response or significant alterations to body composition or mass.

In the open-field test, forced exercisers were significantly less active and entered significantly fewer central squares than voluntary exercisers or sedentary controls. Forced exercise also increased defecation, a measure of emotionality in rodents (Hall, 1934; Sanberg, 1989). These results suggest that long-term forced exercise influences affect-related behaviors. Although we did not directly address the possibility that our forced exercisers would be more likely than wheel runners or sedentary controls to develop learned helpless or depression-like behaviors, this would be interesting to test in future experiments.

Several prior studies have linked exercise to enhanced acquisition in a challenging version of the Morris water maze task (van Praag et al., 1999a, 2005; Vaynman et al., 2004). In contrast, our results do not reflect enhanced performance on this task with either forced or voluntary exercise. It should be noted, however, that the studies showing enhanced performance allowed voluntary exercisers constant access to wheels, whereas, if daily exercise is restricted, there is no enhancement of performance (van Praag et al., 2007). Taken together with our results, the evidence suggests that enhanced performance in a two-trial-per-day water maze paradigm is a consequence of unrestricted voluntary exercise.

Exercise has been shown to enhance proliferation and survival of hippocampal progenitor cells (van Praag et al., 1999a,b; Kim et al., 2002; Vaynman and Gomez-Pinilla, 2005; Uda et al., 2006), so we compared the effects of voluntary and forced exercise on survival of BrdU-labeled progenitor cells in the dentate gyrus. Because of the increased anxiety-like behavior and emotional defecation of the forced exercisers, and prior reports suggesting that forced exercise acutely elevates corticosterone more than wheel running (Ploughman et al., 2005, 2007), we expected survival of BrdU+ cells to be lower in our forced exercisers, compared with voluntary exercisers. We found that both forms of exercise increased survival of BrdU+ cells, although surprisingly, forced exercisers had significantly more surviving cells than voluntary exercisers.

The majority of BrdU + cells also expressed the neuronal marker NeuN, and the percentage of BrdU+ cells that were also NeuN+ did not differ between groups. Thus, exercise did not promote adoption of a neuronal phenotype by newborn cells. Ultimately, however, forced exercise would result in the greatest number of neurons, since the pool of BrdU + cells was largest in that group. Some prior studies have shown that exercise increases the percentage of BrdU+ cells that become neurons (van Praag et al., 1999b; Allen et al., 2001; Steiner et al., 2004), others have not (Farmer et al., 2004; Stranahan et al., 2006). It is interesting to note that the studies that have shown that exercise promotes a neuronal phenotype were performed using mice, whereas those that do not were performed using rats. Because these rat studies also indicate that only a very small percentage of BrdU + cells coexpress the glial markers GFAP or $\mathrm{S} 100 \beta$, we opted to examine colabeling of BrdU with Iba1, a microglial marker. Furthermore, we wanted to ensure that the additional BrdU+ cells present in the forced exercisers were not microglia responding to any adverse conditions in the brain potentially brought on by non-voluntary exercise. We found too few BrdU+/lba1+ cells to enable statistical analysis, however, their scarcity indicates that the additional BrdU+ cells seen in the forced exercisers are not reactive microglia.

It is notable that BrdU+ cells were increased in either of our exercise groups, since the neurogenic effect of exercise has been shown to decline over time (Naylor et al., 2005). In the same study, however, if animals were restricted to $6 \mathrm{~km}$ daily, the increase in BrdU labeling was still present after 3 weeks, which the authors attributed to lower corticosterone levels in the restricted runners compared with those allowed ad libitum access. Our runners were also restricted (although to far less than $6 \mathrm{~km}$ daily) and we examined BrdU labeling after 8 weeks of running, yet the number of BrdU+ cells was still elevated. It has been demonstrated that stimulus withdrawal more effectively drives hippocampal neurogenesis than constant exposure (Kempermann and Gage, 1999). Our results support this idea, since both voluntary and forced exer- 
cisers repeatedly experienced stimulus withdrawal and reintroduction.

The results of the present study suggest that it is unlikely that stress is the critical factor underlying the differential effects of forced and voluntary exercise. We found no differences in corticosterone between groups, although samples were taken during week 8 of exercise, and differences may have been found had we sampled instead during the first week. Although forced exercise does acutely elevate corticosterone to a greater extent than voluntary exercise (Ploughman et al., 2005, 2007), several studies suggest that corticosterone levels return to baseline within several hours after exercise (Stranahan et al., 2006; Ploughman et al., 2007). Furthermore, the HPA axis seems to adapt to chronic running, such that after several weeks, exercise no longer elevates corticosterone (Fediuc et al., 2006). In addition, stress can activate microglia (Nair and Bonneau, 2006; Sugama et al., 2007), yet we found no evidence of enhanced microglial activity in our forced exercisers. We suggest that intensity of exertion may be the most important difference between forced and voluntary exercise. In the present study, intensity of exercise was linked primarily to speed, since neither the forced or voluntary exercisers were running on an incline. Oxygen consumption $\left(\mathrm{VO}_{2 \max }\right)$, a measure of exercise intensity, is tightly correlated to exercise speed. Therefore, since we know the average speed of the voluntary and forced exercisers, we can extrapolate based on the calculations of others (Armstrong et al., 1983; Hoydal et al., 2007) that $\mathrm{VO}_{2 \max }$ was much higher in our voluntary exercisers. Voluntary exercisers ran at higher speed for less time, while forced exercisers ran at lower speed for a longer total period of time (see Fig. 2B, C). The difference in intensity of exertion between the forced and voluntary exercisers may well contribute to the differential effects of these two paradigms on brain and behavior.

Researchers interested in the neurobiology of exercise are rightfully concerned that experimental exercise paradigms should reflect actual human activity. It has been argued that treadmill running, which lasts for a restricted period of time, models human exercise regimens better than voluntary wheel running does (Uda et al., 2006). Treadmill running also forces activity, so one could argue that it reflects the attitude of many human subjects toward exercise. Although it is voluntary, given the often stunning distances covered and speeds maintained by rodents in activity wheels, wheel running may model only those humans that are highly motivated to engage in frequent, sustained physical activity, such as endurance athletes.

\section{CONCLUSION}

In conclusion, it is becoming increasingly apparent that the exercise stimulus itself must be better characterized. The proceedings of a recent workshop held in the interest of advancing the study of the neurobiology of exercise concluded that it is imperative to better understand how exercise itself is influenced by the type, intensity, duration and timing of exertion (Dishman et al., 2006). Our results are a step in this direction, and provide compelling evidence that forced and voluntary exercise are not equivalent, and should not be used interchangeably to study the effects of physical exertion on brain and behavior.

Acknowledgments-The authors thank Ms. Terri Miller for her help exercising the animals, Dr. Michael Grider for assistance with confocal microscopy and imaging, and Drs. Adriana Alcantara and Jack Fletcher for their helpful suggestions on the manuscript. This study was supported by start-up funding for J.L.L. from the University of Houston.

\section{REFERENCES}

Allen DM, van Praag H, Ray J, Weaver Z, Winrow CJ, Carter TA Braquet R, Harrington E, Ried T, Brown KD, Gage FH, Barlow C (2001) Ataxia telangiectasia mutated is essential during adult neurogenesis. Genes Dev 15:554-566.

Ang ET, Dawe GS, Wong PT, Moochhala S, Ng YK (2006) Alterations in spatial learning and memory after forced exercise. Brain Res 1113:186-193.

Ang ET, Gomez-Pinilla F (2007) Potential therapeutic effects of exercise to the brain. Curr Med Chem 14:2564-2571.

Arida RM, Scorza CA, da Silva AV, Scorza FA, Cavalheiro EA (2004) Differential effects of spontaneous versus forced exercise in rats on the staining of parvalbumin-positive neurons in the hippocampal formation. Neurosci Lett 364:135-138.

Armstrong RB, Laughlin MH, Rome L, Taylor CR (1983) Metabolism of rats running up and down an incline. J Appl Physiol 55:518-521.

Bamberg E, Palme R, Meingassner JG (2001) Excretion of corticosteroid metabolites in urine and faeces of rats. Lab Anim 35:307-314.

Brisswalter J, Collardeau M, Rene A (2002) Effects of acute physical exercise characteristics on cognitive performance. Sports Med 32:555-566.

Burghardt PR, Fulk LJ, Hand GA, Wilson MA (2004) The effects of chronic treadmill and wheel running on behavior in rats. Brain Res 1019:84-96.

Carro E, Nunez A, Busiguina S, Torres-Aleman I (2000) Circulating insulin-like growth factor I mediates the effects of exercise on the brain. J Neurosci 20:2926-2933.

Cavigelli SA, Monfort SL, Whitney TK, Mechref YS, Novotny M, McClintock MK (2005) Frequent serial fecal corticoid measures from rats reflect circadian and ovarian corticosterone rhythms. J Endocrinol 184:153-163.

Cotman CW, Berchtold NC, Christie LA (2007) Exercise builds brain health: key roles of growth factor cascades and inflammation. Trends Neurosci 30:464-472.

Dishman RK (1997) Brain monoamines, exercise, and behavioral stress: animal models. Med Sci Sports Exerc 29:63-74.

Dishman RK, Berthoud HR, Booth FW, Cotman CW, Edgerton VR, Fleshner MR, Gandevia SC, Gomez-Pinilla F, Greenwood BN, Hillman CH, Kramer AF, Levin BE, Moran TH, Russo-Neustadt AA JD, Salamone Van Hoomissen JD, Wade CE, York DA, Zigmond MJ (2006) Neurobiology of exercise. Obesity (Silver Spring) 14:345-356.

Dishman RK, Dunn AL, Youngstedt SD, Davis JM, Burgess ML, Wilson SP, Wilson MA (1996) Increased open field locomotion and decreased striatal GABAA binding after activity wheel running Physiol Behav 60:699-705.

Dishman RK, Renner KJ, Youngstedt SD, Reigle TG, Bunnell BN, Burke KA, Yoo HS, Mougey EH, Meyerhoff JL (1997) Activity wheel running reduces escape latency and alters brain monoamine levels after footshock. Brain Res Bull 42:399-406.

Droste SK, Chandramohan Y, Hill LE, Linthorst AC, Reul JM (2007) Voluntary exercise impacts on the rat hypothalamic-pituitary-adrenocortical axis mainly at the adrenal level. Neuroendocrinology 86:26-37. 
Eikelboom R, Mills R (1988) A microanalysis of wheel running in male and female rats. Physiol Behav 43:625-630.

Farmer J, Zhao X, van Praag H, Wodtke K, Gage FH, Christie BR (2004) Effects of voluntary exercise on synaptic plasticity and gene expression in the dentate gyrus of adult male Sprague-Dawley rats in vivo. Neuroscience 124:71-79.

Fediuc S, Campbell JE, Riddell MC (2006) Effect of voluntary wheel running on circadian corticosterone release and on HPA axis responsiveness to restraint stress in Sprague-Dawley rats. J Appl Physiol 100:1867-1875.

Fordyce DE, Farrar RP (1991a) Enhancement of spatial learning in F344 rats by physical activity and related learning-associated alterations in hippocampal and cortical cholinergic functioning. Behav Brain Res 46:123-133.

Fordyce DE, Farrar RP (1991b) Physical activity effects on hippocampal and parietal cortical cholinergic function and spatial learning in F344 rats. Behav Brain Res 43:115-123.

Fulk LJ, Stock HS, Lynn A, Marshall J, Wilson MA, Hand GA (2004) Chronic physical exercise reduces anxiety-like behavior in rats. Int J Sports Med 25:78-82.

Greenwood BN, Foley TE, Day HE, Campisi J, Hammack SH, Campeau S, Maier SF, Fleshner M (2003) Freewheel running prevents learned helplessness/behavioral depression: role of dorsal raphe serotonergic neurons. J Neurosci 23:2889-2898.

Hall C (1934) Emotional behavior in the rat. I. Defecation and urination as measures of individual differences in emotionality. J Comp Psychol 18:385-393.

Hoydal M, Wisloff U, Kemi OJ, Ellingsen O (2007) Running speed and maximal oxygen uptake in rats and mice: practical implications for exercise training. Eur J Cardiovasc Prev Rehabil 14:753-760.

Kamijo K, Nishihira Y, Hatta A, Kaneda T, Kida T, Higashiura T, Kuroiwa K (2004a) Changes in arousal level by differential exercise intensity. Clin Neurophysiol 115:2693-2698.

Kamijo K, Nishihira Y, Hatta A, Kaneda T, Wasaka T, Kida T, Kuroiwa $\mathrm{K}$ (2004b) Differential influences of exercise intensity on information processing in the central nervous system. Eur J Appl Physiol 92:305-311.

Kamijo K, Nishihira Y, Higashiura T, Kuroiwa K (2007) The interactive effect of exercise intensity and task difficulty on human cognitive processing. Int J Psychophysiol 65:114-121.

Kempermann G, Gage FH (1999) Experience-dependent regulation of adult hippocampal neurogenesis: effects of long-term stimulation and stimulus withdrawal. Hippocampus 9:321-332.

Kempermann G, Gast D, Kronenberg G, Yamaguchi M, Gage FH (2003) Early determination and long-term persistence of adultgenerated new neurons in the hippocampus of mice. Development 130:391-399.

Kim SH, Kim HB, Jang MH, Lim BV, Kim YJ, Kim YP, Kim SS, Kim EH, Kim CJ (2002) Treadmill exercise increases cell proliferation without altering of apoptosis in dentate gyrus of Sprague-Dawley rats. Life Sci 71:1331-1340.

Lambert KG, Buckelew SK, Staffiso-Sandoz G, Gaffga S, Carpenter W, Fisher J, Kinsley CH (1998) Activity-stress induces atrophy of apical dendrites of hippocampal pyramidal neurons in male rats. Physiol Behav 65:43-49.

Lambert MI, Van Zyl C, Jaunky R, Lambert EV, Noakes TD (1996) Tests of running performance do not predict subsequent spontaneous running in rats. Physiol Behav 60:171-176.

Leger LA, Taylor AW (1982) The chronic effects of continuous and intermittent running upon lactate dehydrogenase activity of heart, fast and slow twitch muscles in the rat. J Physiol (Paris) 78:499-506.

Masaki T, Nakajima S (2006) Taste aversion in rats induced by forced swimming, voluntary running, forced running, and lithium chloride injection treatments. Physiol Behav 88:411-416.

McMorris T, Collard K, Corbett J, Dicks M, Swain JP (2008) A test of the catecholamines hypothesis for an acute exercise-cognition interaction. Pharmacol Biochem Behav 89:106-115.
Mondon CE, Dolkas CB, Sims C, Reaven GM (1985) Spontaneous running activity in male rats: effect of age. J Appl Physiol 58:1553-1557.

Moraska A, Deak T, Spencer RL, Roth D, Fleshner M (2000) Treadmill running produces both positive and negative physiological adaptations in Sprague-Dawley rats. Am J Physiol Regul Integr Comp Physiol 279:R1321-R1329.

Nair A, Bonneau RH (2006) Stress-induced elevation of glucocorticoids increases microglia proliferation through NMDA receptor activation. J Neuroimmunol 171:72-85.

Narath E, Skalicky M, Viidik A (2001) Voluntary and forced exercise influence the survival and body composition of ageing male rats differently. Exp Gerontol 36:1699-1711.

Naylor AS, Persson Al, Eriksson PS, Jonsdottir IH, Thorlin T (2005) Extended voluntary running inhibits exercise-induced adult hippocampal progenitor proliferation in the spontaneously hypertensive rat. J Neurophysiol 93:2406-2414.

Noble EG, Moraska A, Mazzeo RS, Roth DA, Olsson MC, Moore RL, Fleshner M (1999) Differential expression of stress proteins in rat myocardium after free wheel or treadmill run training. J Appl Physiol 86:1696-1701.

Ploughman M, Granter-Button S, Chernenko G, Attwood Z, Tucker BA, Mearow KM, Corbett D (2007) Exercise intensity influences the temporal profile of growth factors involved in neuronal plasticity following focal ischemia. Brain Res 1150:207-216.

Ploughman M, Granter-Button S, Chernenko G, Tucker BA, Mearow KM, Corbett D (2005) Endurance exercise regimens induce differential effects on brain-derived neurotrophic factor, synapsin-I and insulinlike growth factor I after focal ischemia. Neuroscience 136:991-1001.

Ra SM, Kim H, Jang MH, Shin MC, Lee TH, Lim BV, Kim CJ, Kim EH, Kim KM, Kim SS (2002) Treadmill running and swimming increase cell proliferation in the hippocampal dentate gyrus of rats. Neurosci Lett 333:123-126.

Redila VA, Olson AK, Swann SE, Mohades G, Webber AJ, Weinberg $\mathrm{J}$, Christie BR (2006) Hippocampal cell proliferation is reduced following prenatal ethanol exposure but can be rescued with voluntary exercise. Hippocampus 16:305-311.

Rodnick KJ, Reaven GM, Haskell WL, Sims CR, Mondon CE (1989) Variations in running activity and enzymatic adaptations in voluntary running rats. J Appl Physiol 66:1250-1257.

Russell JC, Epling WF, Pierce D, Amy RM, Boer DP (1987) Induction of voluntary prolonged running by rats. J Appl Physiol 63:2549-2553.

Sanberg PR (1989) Neuroleptic-induced emotional defecation: effects of pimozide and apomorphine. Physiol Behav 46:199-202.

Steiner B, Kronenberg G, Jessberger S, Brandt MD, Reuter K, Kempermann G (2004) Differential regulation of gliogenesis in the context of adult hippocampal neurogenesis in mice. Glia 46:41-52.

Stranahan AM, Khalil D, Gould E (2006) Social isolation delays the positive effects of running on adult neurogenesis. Nat Neurosci 9:526-533.

Sugama S, Fujita M, Hashimoto M, Conti B (2007) Stress induced morphological microglial activation in the rodent brain: involvement of interleukin-18. Neuroscience 146:1388-1399.

Thanos PK, Cavigelli SA, Michaelides M, Olvet DM, Patel U, Diep MN, Volkow ND (2008) A non-invasive method for detecting the metabolic stress response in rodents: Characterization and disruption of the circadian corticosterone rhythm. Physiol Res, in press.

Uda M, Ishido M, Kami K, Masuhara M (2006) Effects of chronic treadmill running on neurogenesis in the dentate gyrus of the hippocampus of adult rat. Brain Res 1104:64-72.

van Praag H, Christie BR, Sejnowski TJ, Gage FH (1999a) Running enhances neurogenesis, learning, and long-term potentiation in mice. Proc Natl Acad Sci U S A 96:13427-13431.

van Praag H, Kempermann G, Gage FH (1999b) Running increases cell proliferation and neurogenesis in the adult mouse dentate gyrus. Nat Neurosci 2:266-270.

van Praag H, Lucero MJ, Yeo GW, Stecker K, Heivand N, Zhao C, Yip E, Afanador M, Schroeter H, Hammerstone J, Gage FH (2007) Plant-derived flavanol (-)epicatechin enhances angiogenesis and retention of spatial memory in mice. J Neurosci 27:5869-5878. 
van Praag H, Shubert T, Zhao C, Gage FH (2005) Exercise enhances learning and hippocampal neurogenesis in aged mice. J Neurosci $25: 8680-8685$

Vaynman S, Gomez-Pinilla F (2005) License to run: exercise impacts functional plasticity in the intact and injured central nervous system by using neurotrophins. Neurorehabil Neural Repair 19:283-295.
Vaynman S, Ying Z, Gomez-Pinilla F (2004) Hippocampal BDNF mediates the efficacy of exercise on synaptic plasticity and cognition. Eur J Neurosci 20:2580-2590.

Winter B, Breitenstein C, Mooren FC, Voelker K, Fobker M, Lechtermann A, Krueger K, Fromme A, Korsukewitz C, Floel A, Knecht S (2007) High impact running improves learning. Neurobiol Learn Mem 87:597-609. 